\title{
Diagnóstico prenatal: Trisomía 13 y holoprosencefalia: Reporte de un caso clínico
}

\author{
Andrés Sarmiento R.*; Jesús A. Berdugo G.**; Guillermo Sánchez B.***
}

\begin{abstract}
RESUMEN: Se presenta un caso de diagnóstico prenatal de trisomía 13 y holoprosencefalia, durante el segundo trimestre de embarazo. Se practica una revisión de la literatura existente, incluyendo criterios de diagnóstico sonográfico y aspectos genéticos asociados. Se insiste en la importancia del diagnóstico sonográfico y genético antenatal, en la determinación de un pronóstico para el manejo ante e intraparto de estos casos.
\end{abstract}

PALABRAS CLAVES: Holoprosencefalia, diagnóstico prenatal, trisomía 13, anomalías cráneo faciales.

SUMMARY: A case of trisomy 13 and holoprosencephaly diagnosed during the second trimester of pregnancy, is presented. The corresponding literature is reviewed, including sonographic diagnostic criteria and associated genetic features. The importance of sonographic and genetic antenatal diagnosis in the determination of a prognosis for the antepartum and intrapartum management for these cases, is emphasized.

KEY WORDS: Holoprosenceplaly, prenatal diagnosis, trisomy 13, brain facial defects.

\section{Introducción}

Desde el advenimiento de la ultrasonografía a la obstetricia, la detección antenatal de anormalidades congénitas se ha convertido en uno de los objetivos básicos de la vigilancia fetal anteparto. El diagnóstico sonográfico de anomalías congénitas ha sido complementado exitosamente con el estudio genético fetal. La combinación de estas dos técnicas ofrece, hoy en día un abordaje completo en términos de diagnóstico prenatal.

A continuación se describe un caso de diagnóstico prenatal de trisomía 13 y holoprosencefalia. En éste, se logró establecer un diagnóstico antenatal anatómico y cromosómico preciso.

\section{Caso clínico:}

Se trata de una paciente de 26 años de edad, primigestante quien cursando embarazo de 19 semanas asistió a control ecográfico rutinario, para confirmar edad gestacional. La paciente había iniciado control prenatal a las 8 semanas de gestación, de un embarazo no complicado. No existían antecedentes familiares de malformaciones congénitas, así como negaba antecedentes tóxico-alérgicos o farmacológicos. Otros antecedentes personales carecían de importancia. Asimismo, no existían antecedentes genéticos en la familia del esposo.

\footnotetext{
* M.D. Ginecobstetra. Sección de ultrasonido ginecobstétrico, Servicio médico - Caja de Previsión Social de Cundinamarca., Santafé de Bogotá. Ultrasonido ginecobstétrico, OBGYN LTDA., Santafé de Bogotá.

** M.D. V.-Genética y reproducción. Servicios Integrales de Reproducción y Genética, Santafé de Bogotá.

*** M.D. Ginecobstetra. Clínica David Restrepo, Santafé de Bogotá
}

Durante el examen sonográfico se detectó un sistema ventricular cerebral dilatado, sin embargo no se pudo definir una anormalidad específica con precisión. Con la sospecha diagnóstica de hidrocefalia incipiente, se solicitó un control ecográfico nivel II, a realizarse 3 semanas más tarde. En este segundo estudio pudo demostrarse un feto de sexo femenino con anormalidades limitadas al examen de la cabeza. En ésta, se demostró una dilatación ventricular marcada de predominio anterior, en la cual proturía el tálamo fusionado. No se identificaron cuernos occipitales aún cuando las estructuras de la fosa posterior lucían normales. El examen facial fetal demostró un hipotelorismo marcado con la presencia de una estructura frontal tubular compatible con una proboscide. (Figura No. 1). No se identificaron anomalías en paladar o boca.

Con una impresión diagnóstica de holoprosencefalia vs. hidranencefalia (??) se decidió complementar el estudio, solicitando un análisis cromosómico prenatal. Para tal efecto, se practicó una amniocentesis a las 22 semanas de gestación.

Un estudio ecográfico realizado tres semanas más tarde (25 semanas), confirmó los hallazgos descritos. Se identificó un holoventrículo anterior con un pequeño remanente de corteza cerebral rechazado hacia la tabla ósea (Figura No. 2). El diámetro biparietal y la circunferencia cefálica confirmaron macrocefalia. A nivel facial se visualizaron hallazgos compatibles con etmocefalia. Nuevamente, no se evidenciaron anormalidades en paladar o boca. Con estos hallazgos sonográficos se hizo un diagnóstico de holoprosencefalia variedad alobar.

Para entonces se obtuvo el resultado del estudio genético, el cual reportó una trisomía 13 por translocación con mosaicismo. (46, XX, -14, + rob (13q; 14q) / $46 \mathrm{XX}$. El 93\% de las metafases analizadas, eran anormales. 

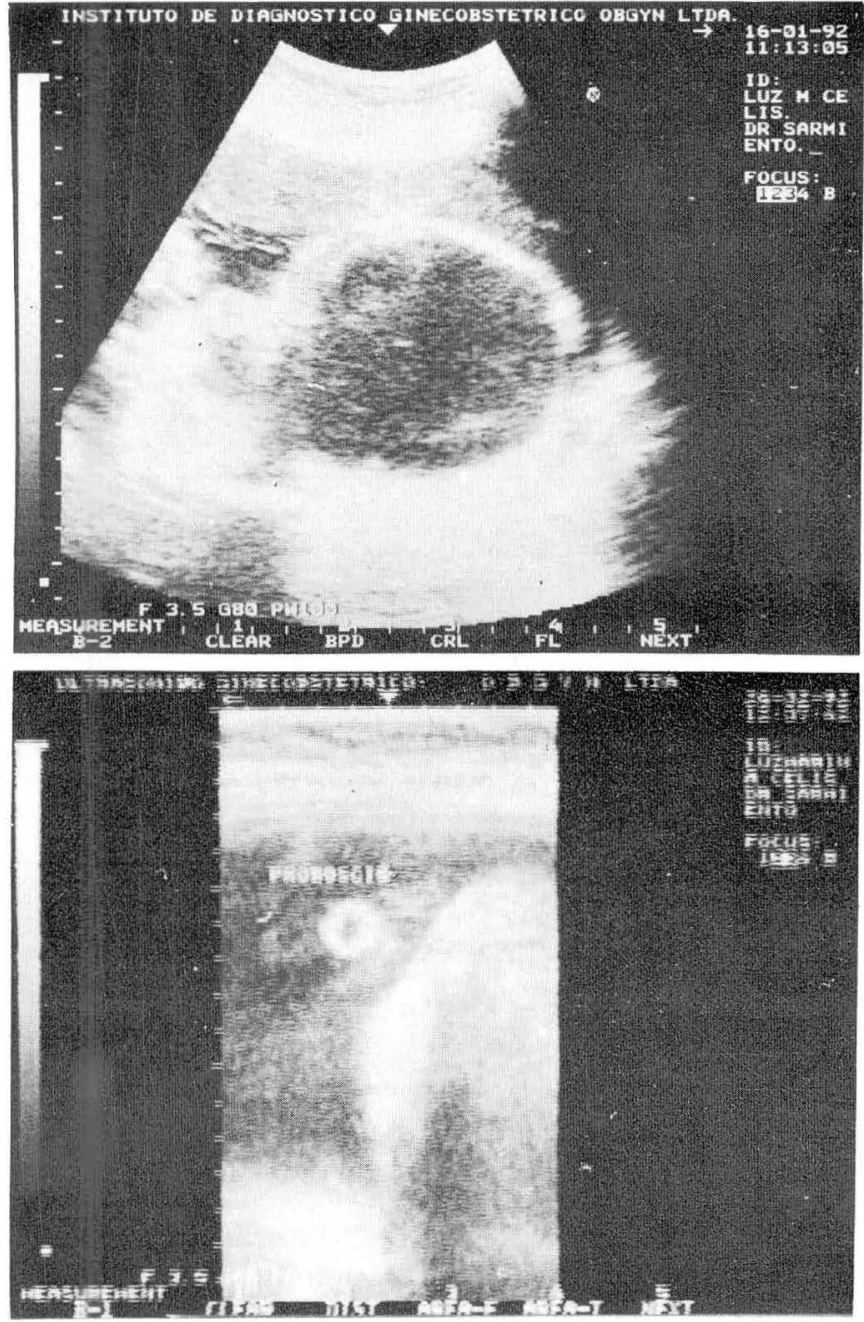

Figura No. 1 (a, b)

ULTRASONIDO DE LA CABEZA FETAL A LAS 25 SEMANAS DE GESTACION

Se visualiza imagen tubular en la region facial, que corresponde a proboscide.

Se procedió a informar a la pareja acerca del pobre pronóstico que conlleva esta anormalidad.

El seguimiento sonográfico no demostró mayores cambios a excepción de la acumulación progresiva de líquido amniótico, hasta consolidarse un polihidramnios franco. A las 34 semanas de embarazo, la paciente inició actividad uterina regular. Ante el pobre pronóstico del embarazo, se decidió no úteroinhibir y permitir el parto. El caso fue llevado a discusión ante un comité médico, decidiéndose desembarazar a la paciente por cesárea ante la evidencia sonográfica de macrocefalia.

La paciente fue llevada a cesárea obteniéndose un RN sexo femenino de $2180 \mathrm{gms}$, talla $45 \mathrm{cms}$, con macrocefalia dada por un perímetro cefálico de $40.2 \mathrm{cms}$. Presentaba hipotelorismo marcado, una proboscide frontal y diástasis de suturas. (Figura No. 3). No existían anomalías de paladar o boca. Además presentaba polidactilia e implantación baja de los pabellones auriculares.
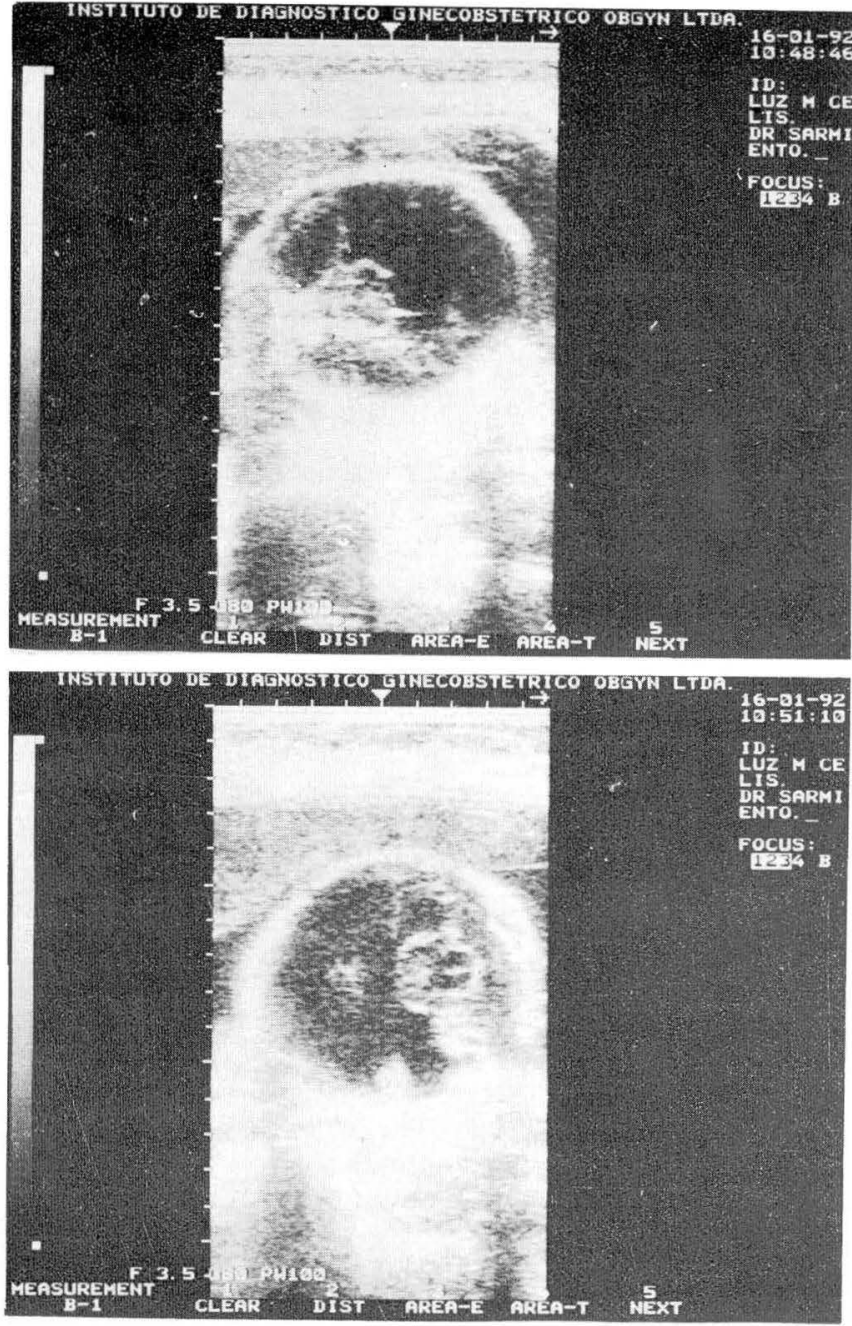

Figura No. 2 (a, b)

CORTE AXIAL DE LA CABEZA FETAL A NIVEL DEL TALAMO Y VENTRICULOS, A LAS 25 SEMANAS DE GESTACION.

Se evidencia un holoventrículo anterior, en el cual protruye el tálamo fusionado. Nótese que existe normalidad en las estructuras de la fosa posterior.

La valoración neonatal determinó un Apgar de 2/10 a los 5 minutos y de $2 / 10$ a los 10 minutos. La recién nacida falleció a los 15 minutos de vida. Se tomaron muestras de sangre de cordón para confirmar el diagnóstico. La familia lamentablemente, no autorizó el estudio anatomopatológico correspondiente.

\section{Discusión}

La holoprosencefalia es una anomalía compleja en el desarrollo cerebral fetal, dada por una falla en la división del prosencéfalo a los 28-32 días de desarrollo embrionario $(1,2)$. Bajo el término holoprosencefalia se engloban un conjunto de anomalías cráneo-faciales de compromiso variable como son ciclopía, cebocefalia, etmocefalia y labio leporino. La entidad puede aparecer como resultado de la alteración de un solo gen o como parte de un síndrome (3).

La incidencia exacta de la holoprosencefalia es difícil de establecer. Un gran número de casos con manifestaciones 

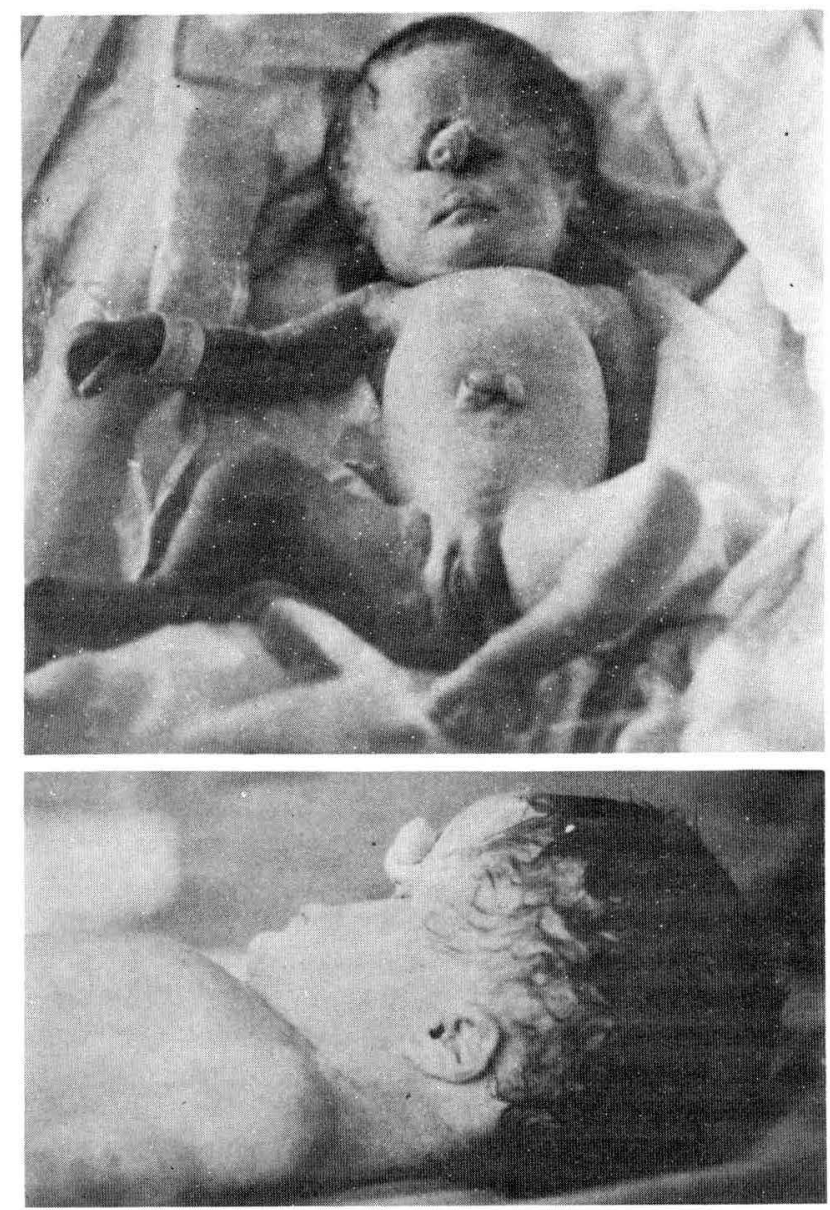

Figura No. $3(\mathrm{a}, \mathrm{b})$

ASPECTOS DE LAS ESTRUCTURAS FACIALES EN EL RECIEN NACIDO.

Se aprecia etmocefalia (hipotelorismo más proboscide), que confirma el diagnóstico sonográfico antenatal (Figura No. 1).

mínimas o sin anormalidades cráneo-faciales evidentes, nunca llegan a diagnosticarse. Sin embargo, se estima que la incidencia puede ser de aproximadamente $0.6 / 1000$ nacidos vivos (1). La incidencia de cebocefalia y labio leporino o de ciclopía como anormalidades aisladas, se ha estimado en $1: 16000$ y $1: 40000$, respectivamente (4).

Los factores etiológicos más comúnmente asociados a holoprosencefalia son el genético y algunos teratógenos. Aún cuando no se ha determinado con exactitud si estos factores son la causa de la holoprosencefalia o su aparición es incidental (5).

Las anormalidades cromosómicas han sido descritas en asocio a holoprosencefalia desde 1963, cuando Miller describió algunas anomalías del sistema nervioso central en relación con trisomías y deleciones $(6,7)$. Las anomalías más frecuentemente asociadas son las trisomías 13, 18 y 21 , los síndromes $18 \mathrm{p}$ - y $13 \mathrm{p}$ - y algunas deleciones.

Se han descrito tendencias hereditarias con patrones autosómico dominante o autosómico recesivo de penetrancia variable. (8-10). Sin embargo, la presencia de holoprosencefalia dentro de alguna entidad clínica es rara. Por ejemplo, 2 de 21 casos en Varadi o síndrome oro-digital-facial (11),
3 de 25 en la secuencia de Digeorge (12), y sólo 1 ó 2 reportes en cada uno de los siguientes síndromes: velocardio facial (13), Majewsky, Pallister-Hall y Smith-Lemli-Opitz (14), Rubinstein-Taybi (15), Walker Warburg (16), Meckel (17), Zellweger (18), y las asociaciones de CHARGE (19), y agnatia-holoprosuicefalia (20).

Los hallazgos citogenéticos más frecuentemente asociados son las alteraciones estructurales y numéricas de los cromosomas 18 y 13. Esta última, es la alteración más comúnmente asociada a holoprosencefalia; hasta un $70 \%$ de fetos afectados presentan este tipo de anormalidad cromosómica (21). Otras anomalías son las duplicaciones de la región distal del brazo corto del cromosoma 3, del brazo largo del cromosoma 7 y deleciones intersticiales del brazo corto del cromosoma $2(22,25,26,28,30)$.

Algunos agentes teratogénicos como etanol, alcaloides, aspirina y radiación han sido propuestos también como posibles factores etiológicos $(4,24,27)$.

Un estudio de 36.380 embriones provenientes de abortos inducidos, encontró una incidencia de holoprosencefalia de 40:10 000. Esta cifra elevada en comparación con la incidencia en nacidos vivos, sugiere que la mayoría de los embriones con esta anomalía están destinados a ser eliminados prenatalmente (23).

La recurrencia de holoprosencefalia en ausencia de aberraciones cromosómicas ha sido estimada en $6 \%$. En presencia de una trisomía primaria, el riesgo puede ser de tan sólo un $1 \%$. En casos donde los padres son portadores de translocaciones, el riesgo puede ser más alto $(4,5)$.

Durante el desarrollo embrionario el prosencéfalo de origen a los hemisferios cerebrales y estructuras diencefálicas, así como participa con el desarrollo normal de las estructuras faciales de la línea media. Un defecto en la actividad del mesénquima precordal sería el responsable del anormal desarrollo prosencefálico y supondría diversos grados de compromiso, con división incompleta de los hemisferios cerebrales y otras estructuras (4). La enfermedad, clínicamente puede manifestarse con macrocefalia o microcefalia.

La holoprosencefalia ha sido clasificada de acuerdo con el grado de alteración prosencefálica, en tres categorías que determinan el compromiso clínico: alobar, lobar y semilobar. La variedad alobar es la forma más severa de holoprosencefalia. Comprende una cavidad ventricular única, con el tálamo fusionado y ausencia de tercer ventrículo, neurohipófisis y tracto óptico. Por lo general no existe compromiso de las estructuras de la fosa posterior ni de tallo cerebral.

En la variedad semilobar persiste un ventrículo único pero con una división parcial de los hemisferios cerebrales en su segmento posterior. Finalmente, en la variedad lobar el compromiso es menor; se aprecian estructuras interhemisféricas definidas aún cuando algunas fusionadas y con ausencia de septum pellucidum.

El compromiso facial de la holoprosencefalia incluye ciertas anomalías clásicas: 1. Ciclopía: comprende monoftalmia, arrinia y presencia de una proboscide. 2. Etmocefalia: hipotelorismo marcado con presencia de proboscide. 3 . Cebocefalia: hipotelorismo con presencia de nariz rudimentaria. 4. Labio leporino: defecto labial de la línea media asociado a hipotelorismo y nariz plana $(1,2,4,29,31)$.

El diagnóstico prenatal sonográfico de holoprosencefalia 
varía en complejidad de acuerdo con la variedad de la misma. La forma alobar, resulta la más accesible al diagnóstico sonográfico por el compromiso marcado y extensivo. Esta se debe sospechar en la presencia de una cavidad ventricular anterior comunicada con un saco dorsal, en el cual protruye el tálamo fusionado. La corteza cerebral se encuentra rechazada cefálicamente por la presencia de la dilatación. Por lo general no existe compromiso de las estructuras dependientes de fosa posterior.

El diagnóstico sonográfico de las formas semilobar y lobar es más complejo; frecuentemente esta última sólo llega a diagnosticarse en una etapa neonatal. En el diagnóstico ecográfico de la variedad semilobar se debe contemplar la presencia de un holoventrículo, con la existencia de una fisura interhemisférica primitiva en la región caudal. La variedad lobar se convierte en la forma de holoprosencefalia más difícil de diagnosticar in-útero, ya que su aspecto sonográfico se acerca a la normalidad. En ella se visualizan los dos hemisferios cerebrales pero existe fusión de estructuras en la región anterior. La no identificación del septum pellucidum (ausente en las tres variedades de la enfermedad) puede ser muy útil en el diagnóstico de esta variedad. Aún cuando se ha reportado el diagnóstico prenatal sonográfico de las tres formas de holoprosencefalia, es frecuente que sea imposible realizar un diagnóstico diferencial preciso.

Las anomalías faciales ya descritas son valiosos recursos sonográficos en el diagnóstico prenatal de holoprosencefalia y en establecer un diagnóstico diferencial con otras entidades.

El diagnóstico diferencial de holoprosencefalia debe hacerse con formas severas de hidrocefalia, con hidranencefalia y agenesia del cuerpo calloso.

El pronóstico de un feto con holoprosencefalia depende de la variedad clínica de la enfermedad. La forma alobar es por lo general fatal. Las variedades semilobar y lobar suponen compromisos variables de disfunción neurológica.

El manejo obstétrico de una paciente con feto holoprosencefálico, debe comprender el estudio genético prenatal dado la alta asociación de la enfermedad con anomalías genéticas. Asimismo, practicar un estudio sonográfico nivel II para fines diagnósticos y en búsqueda de anomalías congénitas asociadas que determinen un pronóstico fetal. Una vez se ha confirmado el diagnóstico, se debe advertir a los padres acerca del mal pronóstico fetal.

En casos de existir evidencia sonográfica y/o genética de compromiso fetal importante, el manejo intraparto debe ser conservador, tendiente a evitar una operación cesárea. En casos de macrocefalia que contraindique el parto vaginal, se recomienda practicar cefalocentesis (4). Asimismo es ideal practicar estudio genético a los progenitores, en búsqueda de patrones hereditarios de la enfermedad.

\section{Conclusión}

En el caso reportado se logró establecer un diagnóstico antenatal, morfológico y genético preciso. Sin embargo, el manejo intraparto no fue consecuente con el pobre pronóstico perinatal que se previó.

La identificación de anormalidades estructurales durante la sonografía fetal rutinaria, debe ser uno de los objetivos básicos del examen obstétrico. Solo un estudio juicioso de la anatomía fetal podrá descartar la presencia de anomalías.

El caso reportado demuestra la importancia del diagnóstico prenatal en la obstetricia moderna. La posibilidad de predecir un resultado final adverso debe ser utilizada en informar a los padres y en poder planear conjuntamente con ellos una estrategia de manejo ante e intraparto.

\section{BIBLIOGRAFIA}

1. Harrison M. The Unborn patient. 2nd Ed. San Francisco, California. WB Saunders Co. 1991; 410-413.

2. Cohen MM. An update on the holoprosencephalic disorders J. Ped. 1982; 101: 865-869.

3. Webster WS., Lipson AH., Sulik KK. Interference with gastrulation during the third week of pregnancy as a cause of some facial abnormalities and CNS defects. Am. J. Med. Genet. 1988; 31: 505-512.

4. Romero R. Prenatal Diagnosis of Congenital Anomalies. 1a. Ed. New Haven Ct, Appleton-Lange 1988; 59-65.

5. Munke M. Clinical, cytogenetic and molecular approaches to the genetic heterogeneity of holoprosencephaly. Am. J. Med. Genet. 1989; 34: 237-245.

6. Miller JQ., Pickard EH., Alkan MK., Warner S. et al. A specific brain defect (archinencephaly) in 13-15 trisomi. N. Engl. J. Med. 1963; 26: 120-124.

7. De Groucho J., Lamy M., Thieffry S., Arthuis M. Dysmorphie complexe avec oligophrénie: Deletion des bras courts d'un chromosome 17-18. C.R. Acad. Sci. 1963; 256: 1028-1029.

8. De Meyer W., Zeman W., Palmer C. Familiar holoprosencephaly (arihinencephaly) with median cleft lip and palate. Neurology 1963; 13: 913-918.

9. Johnson VP. Holoprosencephaly: a developmental field defect. Am. J. Gen. 1989; 34: 255-257.

10. Mc Donald DM., Menuti Godwin DC., Kistenmacher MN., Zackai EH. Holoprosencephaly a heterogeneus disorder. Am. J. Med. Genet. 1988; Suppl 4.
11. Varadi B., Szabo L., Papp Z. Syndrome of polydactily, cleft lip/palate or lingual lump, and psychomotor retardation in endogamic gypsies. J. Med. Genet. 1980; 17: 119-122.

12. Conley ME., Beckwith J., Mancver JFK., Tenckhoff L. The spectrum of di George Syndrome. Clin. Genet. 1985; 27: 408-410.

13. Wraight JE., Super M., Watson JH., Phillips M. Velocardio-facial syndrome presenting as holoprosencephaly. Clin. Genet. 1985; 27 : 408-410.

14. Donnai D., Burn J., Hughes H. Smith-Lemli-Optiz syndromes: Do they include the Pallister-Hall syndrome. Am. J. Med. Genet. 1987; 28: 741-743.

15. De Meyer, De Grouchy J., Tourliau C. Clinical Atlas of Human Chromosomes. Holoprosencephaly (cyclopia-archinenceophaly). 1 Ed. New York, John Wiley and Sons. 1977; 308-313.

16. Dobyns WB., Pagon RA., Armstrong DM., Curry CJ., Greenberg F. et al. Diagnostic criteria for walker-Warburg sindrome. Am. J. Med. Genet. 1989; 32: 195-210.

17. Bergma D., Optiz JM., Howe JJ. The clinical delineation of birth defects and malformation syndromes. The Meckel syndrome (Dysencephalia Splanchocystica: the Gruber syndrome). 1 ed. Baltimore, The John Hopkins Medical Institutions for the national foundation March of Dimes. 1969: D.B. OAS V(2), 167-179.

18. Bergma D., Optiz JM., Zurhein GM., Vitale L., Shahidi NT., Howe JJ. et al. The clinical delineation of birth defects and malformation syndromes. The Zellweger syndrome (cerebro-hepato-renal syndrome). 
1 ed. Baltimore, The John Hopkins medical Institutions for the National Fundation - March of dimes. 1969: D.B. OAS V(2), 144-158.

19. Torriello HV. Arhinencephaly field defect. Am. J. Med. Genet. 1986; 2: 73-76.

20. Pauli RM., Pettersen JC., Arya S., Gilbert EF. Familial Agnathia Holoprosencephaly. Am. J. Med. Genet. 1983; 14: 677-698.

21. Warkany J., Passarge E., Smith LB. Congenital malformations in autosomal trisomy syndromes. Am. J. Dis. Child. 1966; 112: 502-517.

22. Munke M., Emmanuel DS., Zanckai H. Holoprosencephaly: Association with intersticial deletion of the $2 p$ and review of the citogenetic literature. Am. J. Med. Genet. 1988; 30: 929-938.

23. Matsunaga E., Shiota K. Holoprosencephaly in human embryos: Epidemiologic studies in 150 cases. Teratology, 1977; 16:261-277.

24. Siebert JR., Astley S. Holoprosencephaly in a fetal Macaque following weekly exposure to ethanol. Teratology, 1991; 44: 29-36.

25. Berry SM. Fetal Holoprosencephaly: Associated Malformations and Chromosomal Defects. Fetal Diagnosis Therapy. 1990; 5: 92-99.
26. Hatziioannou AG. Familial holoprosencephaly associated with a translocation breakpoint at chromosomal position 7q36. Amer. J. Med Genetics. 1991; 40: 201-205.

27. Ronen GM. Holoprosencephaly as a possible embryonic alcohol effect. Amer. J. Med Genet. 1991; 40: 151-154.

28. Verloes A. Holo-Polydatyly ("pesudotrisomy 13") syndrome: a syndrome with features of hydrolethalus and Smith-Lemli-Opitz syndromes. A Collaborative multicentre study. J. Med. Genet. 1991; 28: 297-303.

29. Souza JP. An anatomic comparison of cebocephaly and ethmocephaly. Teratology. 1990; 42: 347-357.

30. Isada NB. Trisomy 22 with Holoprosencephaly: a clinicopathologic study, Teratology. 1990; 42: 333-336.

31. Toma P. Holoprosencephaly: Prenatal diagnosis by sonography and magnetic resonance imaging. Prenatal Diagnosis 1990; 10: 429-436.

\section{Eventos}

XVI CONGRESO LATINOAMERICANO DE OBSTETRICIA Y GINECOLOGIA

Centro de Convenciones de Atlapa - Panamá Noviembre de 1993

XVI CURSO BIENAL INTERNACIONAL DE ACTUALIZACION EN OBSTETRICIA

Y GINECOLOGIA 50 AÑOS SCOG

Santafé de Bogotá, Febrero 28, 1, 2 y 3 de marzo de 1993

\section{CONGRESO COLOMBIANO DE OBSTETRICIA Y GINECOLOGIA}

Santafé de Bogotá, Marzo de 1994 